\title{
App móvil para el registro de sismos en Ecuador mediante la geolocalización
}

\author{
Fecha de recepción: 11-01-2021 • Fecha de aceptación: 15-03-2021 • Fecha de publicación:10-06-2021
}

\author{
Josselyn Silvana Rodríguez Mejía \\ Investigador independiente, Ecuador \\ joss_silvana1995@hotmail.com \\ https://orcid.org/0000-0003-4535-8084
}

\section{RESUMEN}

El Instituto Geofísico de la Escuela Politécnica Nacional menciona que el Ecuador está cerca de la zona donde convergen las placas Nazca y Sudamericana, lo que determina una importante actividad sísmica. Este trabajo investigativo describe la creación de una aplicación móvil que como resultado agiliza el registro de haber sentido el movimiento telúrico tomando la ubicación exacta mediante geolocalización, además de datos relevantes como fecha, hora y qué tan fuerte se sintió el sismo. Para llevar a cabo el sistema planteado se utilizó la metodología Scrum, en la cual definieron roles, historias de usuario y producto. Para probar el correcto funcionamiento se realizaron pruebas en dispositivos móviles físicos para la aplicación móvil y la página de administración de sismos.

\section{PALABRAS CLAVE: sismos, instituto geofísico, sistema móvil, geolocalización.}

\section{ABSTRACT}

The Geophysical Institute of the National Polytechnic School mentions that Ecuador is close to the area where the Nazca and South American plates converge, which determines an important seismic activity. This research work describes the creation of a mobile application that as a result speeds up the registration of having felt the telluric movement by taking the exact location through geolocation, in addition to relevant data such as date, time and how strong the earthquake was felt. To carry out the proposed system, the Scrum methodology was used, in which roles, user stories and product 
were defined. To test the correct operation, tests were performed on physical mobile devices for the mobile application and the earthquake management page.

KEYWORDS: earthquakes, geophysical institute, mobile development, geolocation. 


\section{Introducción}

En los últimos años el uso de sistemas móviles (teléfonos móviles, tablets, cámaras digitales, etc.) se ha incrementado de manera considerable, y por ende, el desarrollo de aplicaciones que faciliten la vida diaria de las personas, aplicaciones como apoyo al uso de energía eléctrica (Toasa et al., 2017), aplicaciones móviles para la gestión de procesos industriales (Reyes et al., 2019), para potenciar el turismo (Silva et al., 2018), y se puede enumerar un sinfín más de apps.

En el contexto europeo se desarrollan aplicaciones móviles (Mascort-Albea et al., 2019) para las cuales se proporciona un conjunto de protocolos a seguir en la ocurrencia de un desastre natural, contando con el apoyo de distintas aplicaciones móviles referentes a desastres como los sismos que muestran información, en González et al. (2016) se mencionan dos sistemas, uno web, que es un visualizador, y uno móvil para el envío de notificaciones. Esto sirvió como base para poder desarrollar este trabajo: el desarrollo de una app móvil que permita gestionar los sismos en el contexto ecuatoriano.

Luego de una gran catástrofe originada por la naturaleza, se genera graves consecuencias en la sociedad que puede demorar meses, e incluso años en recuperarse y volver a la normalidad, una de las principales acciones que se hace es atender a las personas heridas o fallecidas, el restablecimiento de las actividades en los lugares afectados en la sociedad, entre otras. Para esto se necesita registros de las personas y lugares donde sintieron el movimiento telúrico, lo cual ayudaría a los rescatistas a llegar a las zonas realmente más afectadas por dicho evento.

Actualmente el Instituto Geofísico de Ecuador (2021) cuenta con un formulario para registrar si se sintió el sismo en un determinado lugar, las personas lo pueden llenar utilizando un computador o dispositivo móvil completando varias preguntas como: el código generado por el instituto geofísico, el lugar, hora, provincia, intensidad del sismo, entre otras, esto lo hace un formulario extenso, considerando que una de las recomendaciones para la creación de estos es solicitar datos indispensables, lo que conlleva a la de reducción de los campos solicitados en un formulario, con lo planteado se conseguirá ahorrar tiempo a los usuarios e incrementar la tasa de éxito de finalización de la tarea, y la conversión, si se trata de un formulario transaccional.

Al contar con este sistema móvil se tendrán registros de los usuarios en las zonas más afectadas, lo cual permitirá al Estado o instituciones encargadas de los rescates llegar a las zonas realmente afectadas de manera oportuna, teniendo así la posibilidad de salvar más vidas, ya que los registros se harán al instante en un tiempo mínimo. Para agilizar dicho registro se pretende incorporar el API de geolocalización (APIs de Geolocalización, s.f), permitiendo de esta manera obtener el lugar exacto del usuario, así como también la hora y fecha que se tomará del dispositivo que el usuario este utilizando, además de incorporar también las notificaciones para que el usuario se entere de cuando sucede un movimiento telúrico. 


\section{Metodología}

El proceso en el desarrollo de este trabajo se realizaró inicialmente con la definición del proceso actual, el cual se pretende automatizar.

La Figura 1 muestra el proceso con el cual trabaja el Instituto Geofísico - Escuela Politécnica Nacional en el área de registro de los usuarios que sintieron un sismo, lo cual se quiere simplificar con la implementación del sistema.

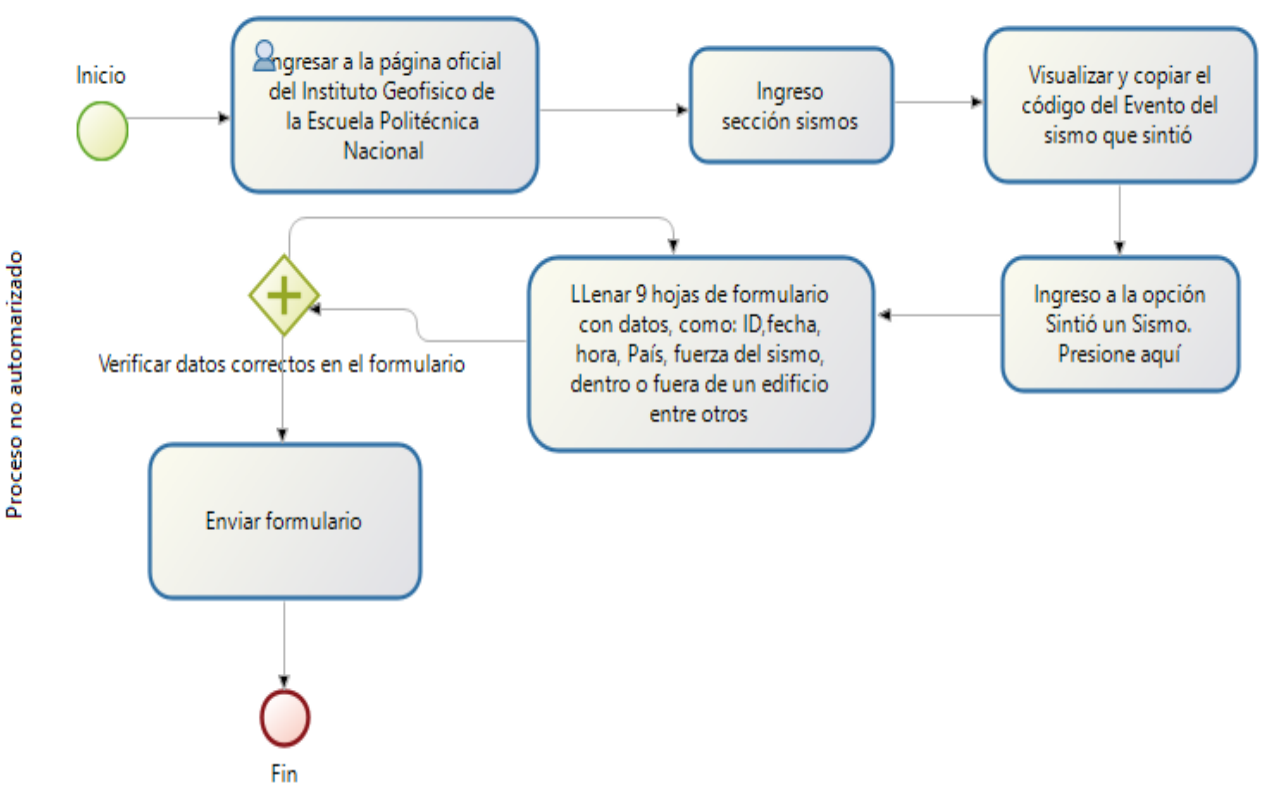

Figura 1. Proceso Actual - No automatizado

Fuente: elaboración propia

En la Figura 2 se describe la forma que se va a seguir para automatizar el proceso, cabe resaltar que se toman en cuenta los departamentos involucrados en cada fase del proceso. 


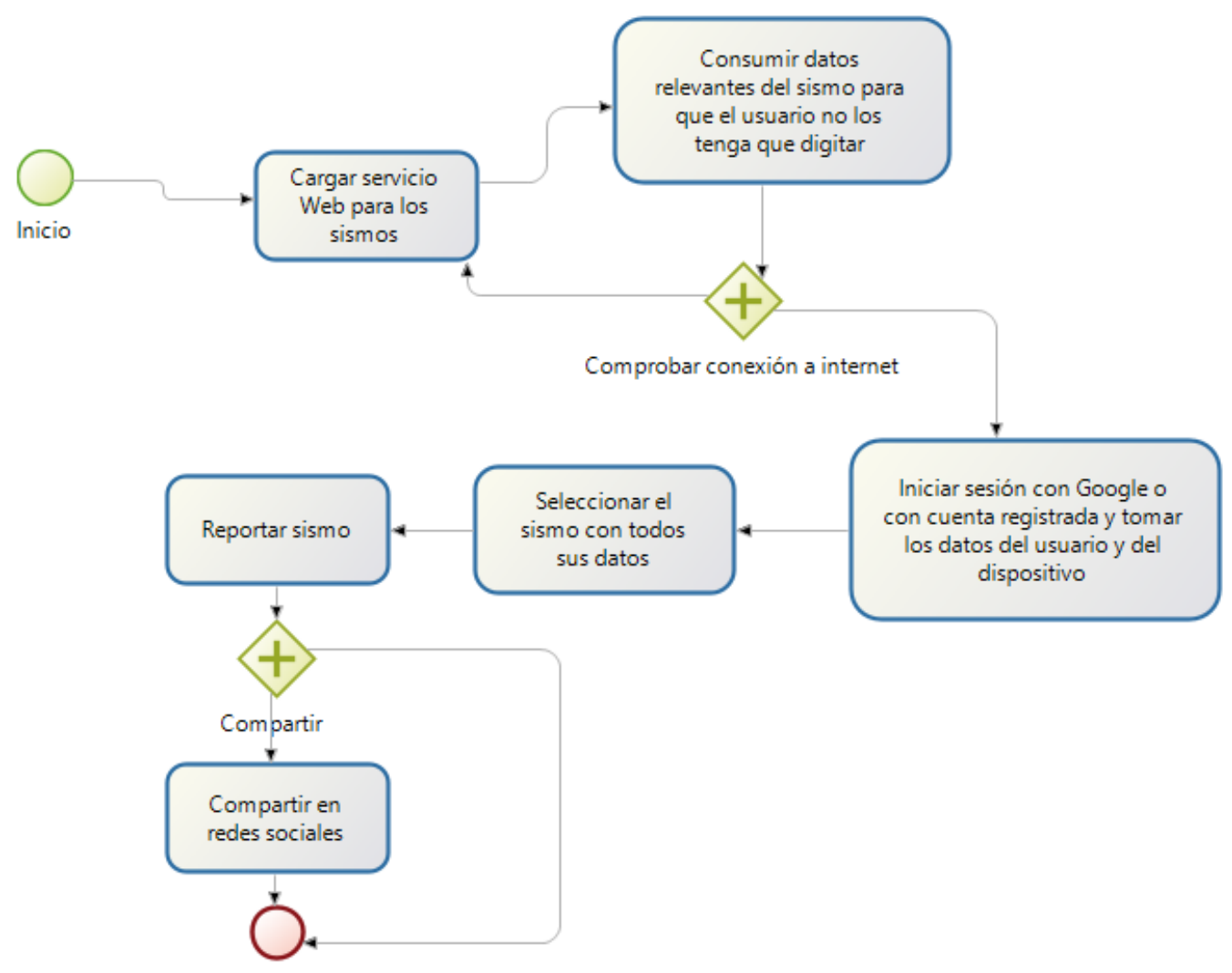

Figura 2. Proceso Automatizado

Fuente: elaboración propia

Para llevar a cabo el sistema se utilizó la metodología Scrum (proyectosagiles, s.f), en la cual se ha definido roles, historias de usuario y producto backlog y sprint backlog.

En la siguiente Tabla 1 se evidencian los roles para el desarrollo de este trabajo.

Tabla 1.

Equipo de trabajo y roles

\begin{tabular}{|l|l|}
\hline Personas / Entidad & Rol \\
\hline Universidad Tecnológica Israel & Product Owner \\
\hline Mg. Renato Toasa & Scrum Master \\
\hline Josselyn Rodríguez & Development Team \\
\hline
\end{tabular}

Fuente: elaboración propia

En la Tabla 2 se muestra cómo será la historia de usuario para reportar un sismo. 
Tabla 2.

Historia de usuario para reportar sismo sentido

\begin{tabular}{|l|l|}
\hline \multicolumn{2}{|c|}{ HISTORIA DE USUARIO } \\
\hline Número: 8 & Nombre: Reportar sismo sentido \\
\hline Usuario: Usuario final & Riesgo en Desarrollo: Alta \\
\hline Prioridad en negocio: Alta & Iteración asignada: 1 \\
\hline $\begin{array}{l}\text { Descripción: Los usuarios registrados podrán reportar si sintió un sismo en la aplicación móvil. } \\
\text { Debe seleccionar un sismo de la lista y colocar datos como que tan fuerte sintió el sismo, si se encuentra } \\
\text { dentro o fuera de un inmueble y los demás serán tomados del dispositivo móvil. }\end{array}$ \\
\hline $\begin{array}{l}\text { Observación: El dispositivo móvil debe tener acceso a Internet, caso contrario se mostrará un mensaje al } \\
\text { usuario para que se conecte a una red. }\end{array}$ \\
\hline
\end{tabular}

Fuente: elaboración propia

En la siguiente Tabla 3 se observa el product backlog.

Tabla 3.

Producto Backlog

\begin{tabular}{|l|l|l|l|}
\hline N. Historia & Enunciado Product Backlog & Sprint & Estado \\
\hline 1 & Iniciar sesión en la página web del servicio web & 1 & Terminado \\
\hline 2 & Insertar sismos en la página del servicio web & 1 & Terminado \\
\hline 3 & Modificar sismos en la página del servicio web & 1 & Terminado \\
\hline 4 & Eliminar sismos en la página del servicio web & 1 & Terminado \\
\hline 5 & Iniciar sesión en la aplicación móvil & 1 & Terminado \\
\hline 6 & Registrar usuarios finales & 1 & Terminado \\
\hline 7 & Modificar datos de usuarios finales & 1 & Terminado \\
\hline 8 & Reportar sismo sentido & 1 & Terminado \\
\hline 9 & Compartir en redes sociales el sismo sentido & 1 & Terminado \\
\hline 10 & Notificar nuevo registro de sismo & 1 & Terminado \\
\hline
\end{tabular}

Fuente: elaboración propia

\section{Mientras que en la Tabla 4 se puede ver el sprint backlog}


Tabla 4.

Sprint backlog

\begin{tabular}{|l|l|l|l|}
\hline \multicolumn{3}{|l|}{$\begin{array}{l}\text { Entregable: Acceder a la aplicación móvil para registrar usuarios con un formulario en el cual solicita: } \\
\text { email, nombre, apellido y contraseña del usuario. }\end{array}$} & $\begin{array}{l}\text { Tiempo } \\
\text { estimado } \\
\text { (Horas) }\end{array}$ \\
\hline $\begin{array}{l}\text { Identificador del } \\
\text { product backlog }\end{array}$ & $\begin{array}{l}\text { Enunciado de product } \\
\text { backlog }\end{array}$ & Tareas & 30 \\
\hline 6 & $\begin{array}{l}\text { Registrar usuarios } \\
\text { finales }\end{array}$ & $\begin{array}{l}\text { Diseño de la interfaz de usuario para } \\
\text { registrar usuarios. }\end{array}$ & \\
& & $\begin{array}{l}\text { Definición de función para registrar } \\
\text { usuarios. }\end{array}$ & \\
& $\begin{array}{l}\text { Integración de firebase para uso de } \\
\text { Database RealTime. }\end{array}$ \\
& $\begin{array}{l}\text { Elaboración de validaciones de los } \\
\text { campos de registro. }\end{array}$ & \\
\cline { 3 - 3 } & Pruebas con el usuario \\
\hline
\end{tabular}

Fuente: elaboración propia

Luego de la recolección de la información, se obtuvo los requisitos para plantear los requerimientos funcionales y no funcionales (López, 2001) de la aplicación móvil y de la página web que se muestran a continuación:

\subsection{Funcionales para la página web}

Considerando que solo es un prototipo de la aplicación móvil, se implementó un propio servicio de consumo de información, considerando que los datos serán tomados de la página oficial del Instituto Geofísico de la Escuela Politécnica Nacional para fines académicos.

- RF01: el usuario debe iniciar sesión con las credenciales dadas por el desarrollador, ya que será un único usuario administrador.

- RF02: se permitirá el registro, al igual que su modificación de información y borrado de los mismos.

- RF03: la página listará los sismos registrados al igual que los usuarios que reporten que sintieron un sismo.

\subsection{Funcionales para la aplicación móvil}

- RF04: se permitirá el registro de usuarios con un formulario dado o mediante el uso de la cuenta del Google.

- RF05: el inicio de sesión será mediante correo y contraseña o Google, con la validación de dichas credenciales. 
- RF06: la aplicación móvil permitirá que el usuario actualice sus datos, tales como el nombre, apellido y contraseña.

- RF07: se listará los sismos registrados en la página web y el usuario podrá reportar si ha sentido el sismo seleccionado donde se registra la ubicación actual del usuario, hora y fecha, qué tan fuerte lo sintió y si se encuentra dentro o fuera de una edificación.

- RF08: se mostrará una lista de los sismos reportados por el usuario.

- F09: podrá compartir el sismo en redes sociales como WhatsApp, Twitter o Facebook.

- RF10: se notificará al usuario mediante su dispositivo móvil si se ha registrado algún sismo en la página web.

\subsection{No funcionales}

- RNF01: tanto la página web como la aplicación móvil deben tener interfaces graficas bien formadas y legibles.

- RNF02: la página web de administración debe poseer un diseño adaptativo para la adecuada visualización en múltiples computadores.

- RNF03: diseñar de manera simple y funcional, con variables legibles y claros, carpetas ordenadas donde se acumule los contenidos siguiendo la modelo vista controlador.

- RNF04: facilitar el mantenimiento en cuanto al cumplimiento de estándares, uso de guías y patrones, documentación y de navegabilidad intuitiva.

- RNF05: el tiempo de respuesta para los usuarios de la aplicación móvil inmediata no debe superar los 3 segundos.

- RNF06: la contraseña de los usuarios se guarda encriptada en la base de datos.

- RNF07: usar navegadores web como Google Chrome y Mozilla Firefox para la página web y sistema operativo Android para la aplicación móvil.

- RNF08: utilizar base de datos no relacional.

Para el almacenamiento de control de la versión del código fuente se utilizó GitHub (Blischak et al., 2016) que está creada para que los desarrolladores suban el código de sus aplicaciones y herramientas, y que como usuario, pueda descargar la aplicación, sino también entrar a su perfil para leer sobre la contribución que hizo en el proyecto, por otro lado, Git diseñado por Linus Torvalds. 


\subsection{Estándares de programación}

Los estándares de programación que se utilizaron fueron:

- Estilo de escritura: lowerCamelCase (Binkley et al., 2009), para todo el desarrollo del proyecto.

- Lenguaje de programación: Java para la aplicación móvil y TypeScript para la página web.

- Herramienta de desarrollo: Android Studio (Android Developers, 2020) para la aplicación móvil y Angular con Bootstrapc (Angular Directives for Bootstrap, s.f.) para la página web.

- Base de datos no relacional: Firebase (Moroney, 2017), las tablas son en plural y comienzan con minúsculas.

- Metodología: Scrum

\section{Resultados}

El resultado de este proyecto se validará mediante las pruebas al sistema, con estas se verificará qué tanto la aplicación móvil y la página web cumple con las especificaciones planteadas en el estudio, para considerar aprobado las funcionales de cada módulo. A continuación, en la Tabla 5 se coloca un ejemplo de las pruebas funcionales:

Tabla 5.

Prueba de aceptación de notificaciones de sismos

\begin{tabular}{|l|l|l|l|l|}
\hline Título & Contexto & Evento & Resultado & Evaluación \\
\hline Notificación & En el caso & Cuando el administrador & La aplicación & Aprobada \\
de nuevo & que el & registre un sismo nuevo & móvil mostrará \\
sismo & administrador & & $\begin{array}{l}\text { unotificación } \\
\text { con los datos del } \\
\text { sismo nuevo }\end{array}$ & \\
& registre un & & & \\
& nuevo sismo & & \\
\hline
\end{tabular}

Fuente: elaboración propia

Finalmente, se muestra en las siguientes figuras que la aplicación móvil fue probada y usada por usuarios en dispositivos reales, dando por hecho que no se reportó ningún inconveniente en el uso de la misma. 


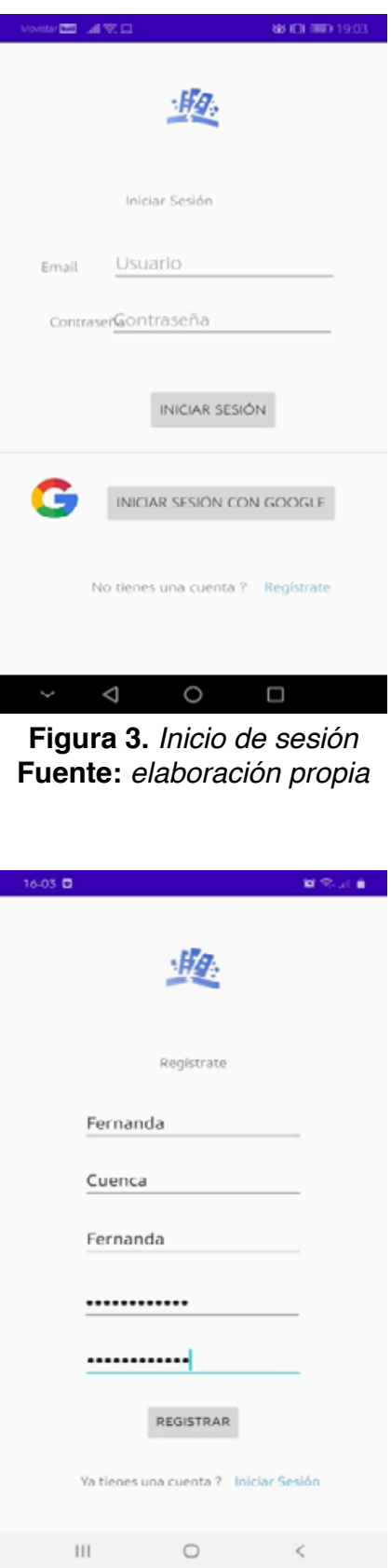

Figura 5. Registro de usuario Fuente: elaboración propia

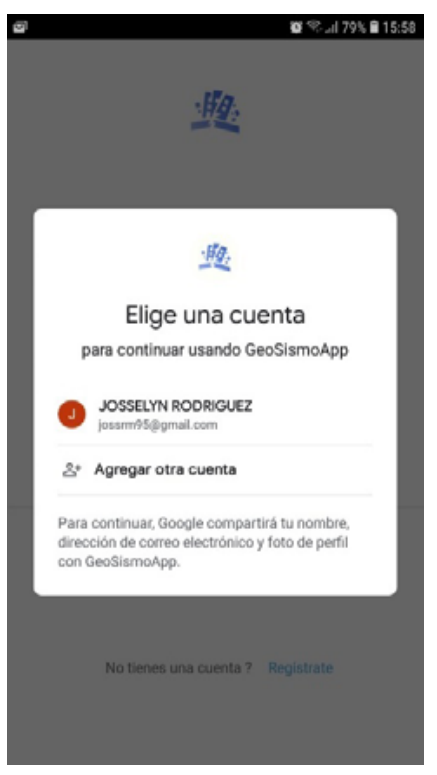

Figura 4. Inicio de sesión con Google Fuente: elaboración propia

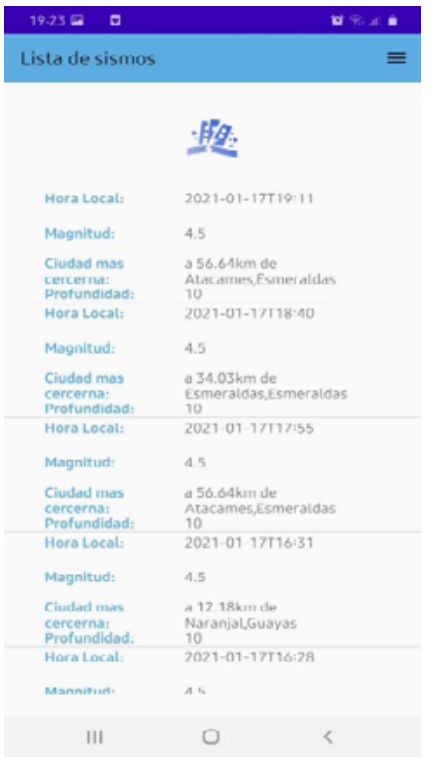

Figura 6. Lista de sismos Fuente: elaboración propia 


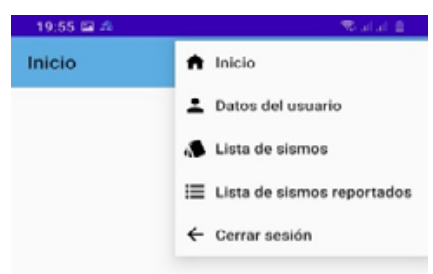

Bienvenid@:

Cristhian Camacho

Figura 7. Pantalla de inicio

Fuente: elaboración propia

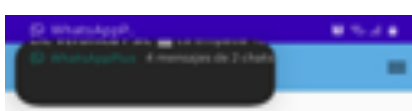

A

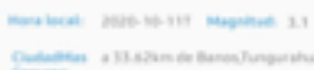

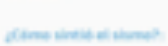

Lene ineness percoster

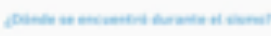

Oentes de une esticasien

unesis

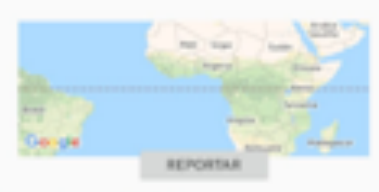

$\boldsymbol{0} \mathrm{c}$

Figura 8. Reportar sismo

Fuente: elaboración propia

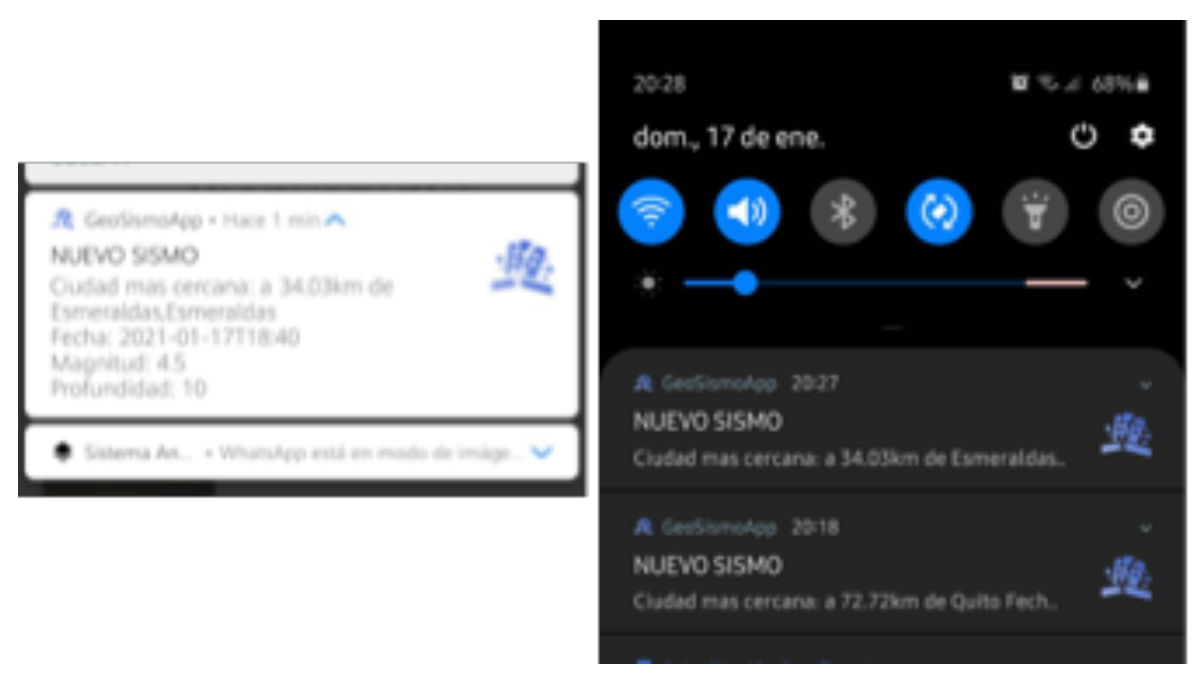

Figura 9. Notificaciones de registro de nuevo sismo

Fuente: elaboración propia 

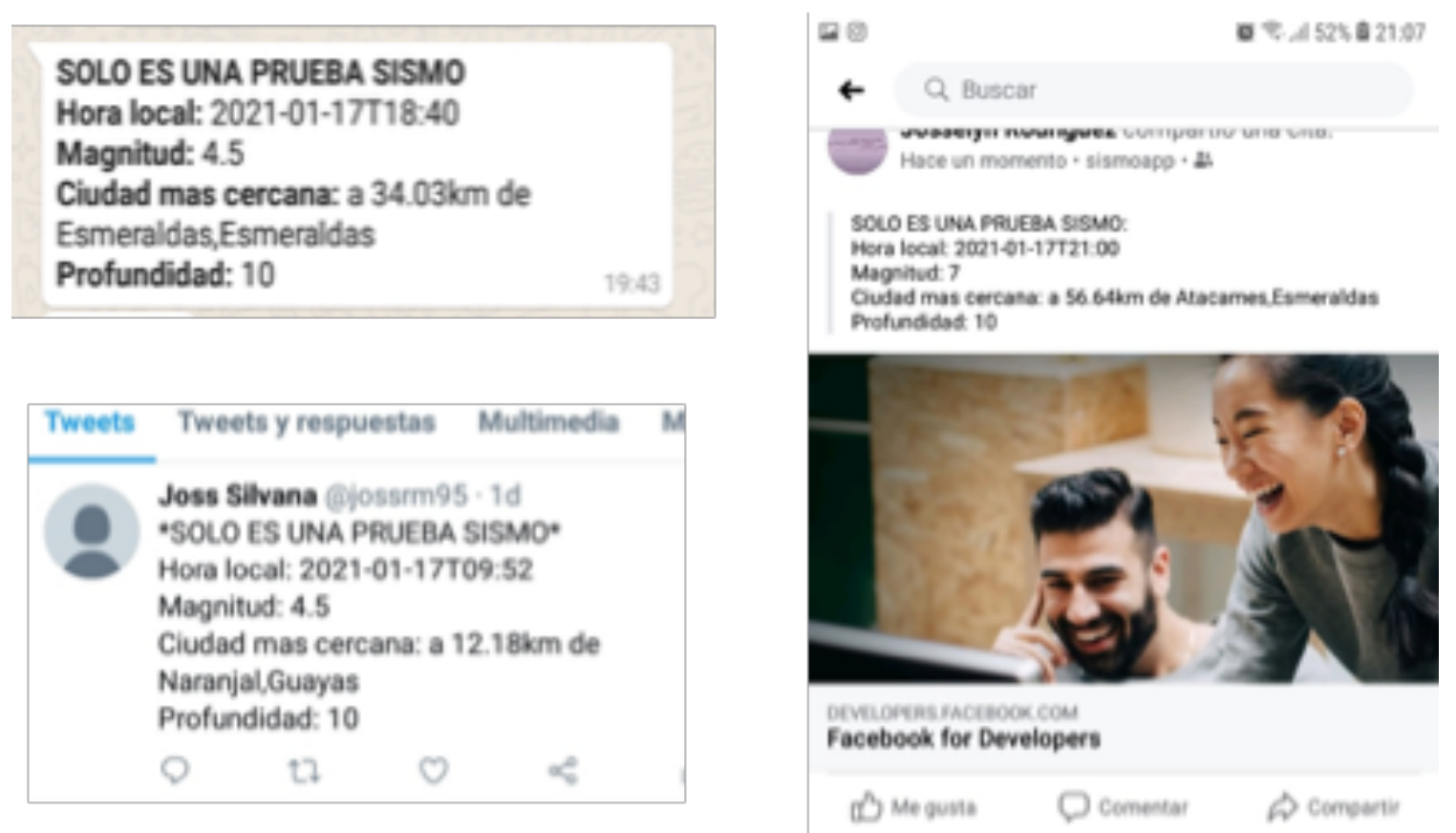

Figura 10. Compartir en redes sociales de WhatsApp, Twitter y Facebook

Fuente: elaboración propia

\section{Conclusiones}

Como parte de conclusiones se evidenció que si bien el Instituto Geofísico - EPN brinda el servicio de reportar si se ha sentido un sismo en el momento que ocurra dicho movimiento telúrico, se determinó que el mismo es muy largo, incluyendo entre 12 y 17 preguntas, esto invalida el reportar catástrofe natural.

La utilización de AngularJS junto con Nodejs y Firebase como servidor para la aplicación móvil y página web administrativa proporciona una capa de abstracción en el consumo de información y los procesos CRUD.

La aplicación móvil que fue desarrollada en el artículo con Android Studio, utilizando el lenguaje Java, permite una reducción de tiempo en el desarrollo ya que se trabajó de forma nativa para el sistema operativo Android. La utilización de estas herramientas facilitó a su vez el alcance de los objetivos propuestos en el proyecto, además, con la metodología ágil Scrum se facilitó el llevar a cabo entregas del proyecto en los tiempos establecidos.

Para probar el correcto funcionamiento se realizaron pruebas en dispositivos móviles físicos para la aplicación móvil y la página de administración de sismos, también se realizaron pruebas de aceptación de software para comprobar que se cumplió con todos los requisitos propuestos en las historias de usuario. 


\section{Referencias}

Angular directives for Bootstrap. (s.f). https://angular-ui.github.io/bootstrap/

Android Developers. (2020). Developer. https://developer.android.com/?hl=es

APIs de geolocalización (s.f). Google Maps Platform I Google Cloud. https://cloud.google.com/maps-platfor$\underline{\mathrm{m} ? \mathrm{hl}=\mathrm{es}}$

Binkley, D., Davis, M., Lawrie, D., \& Morrell, C. (2009). To camelcase or under-score. IEEE 17th International Conference on Program Comprehension, 158-167. https://doi.org/10.1109/ICPC.2009.5090039

Blischak, J. D., Davenport, E. R., \& Wilson, G. (2016). A Quick Introduction to Version Control with Git and GitHub. PLoS Computational Biology, 12(1), e1004668. https://doi.org/10.1371/journal.pcbi.1004668

González, C., Martín, E., Antón, R., \& Zomoza, J. F. V. (2016). Información sobre terremotos: visualizador y APP de sismología. Topografía y cartografía: Revista del Ilustre Colegio Oficial de Ingenieros Técnicos en Topografía, 34(172), 37-40. https://dialnet.unirioja.es/servlet/articulo?codigo=7446498

Instituto Geofísico. (2021). Informes de los últimos sismos. IGEPN https://www.igepn.edu.ec/ultimos-sismos

López, O., Laguna, M. Á., \& Marqués, J. M. (2001). Reutilización del Software a partir de Requisitos Funcionales en el Modelo de Mecano: Comparación de Escenarios. Actas de IDEAS 2001.

Mascort-Albea, Canivell, Jaramillo-Morilla, Romero-Hernández, Ruiz-Jaramillo, \& Soriano-Cuesta. (2019). Action protocols for seismic evaluation of structures and damage restoration of residential buildings in Andalusia (Spain): "IT-Sismo" APP. Buildings, 9(5), 104. https://doi.org/10.3390/buildings9050104

Moroney, L. (2017). Firebase Cloud Messaging. In The Definitive Guide to Firebase (pp. 163-188). Apress. https://doi.org/10.1007/978-1-4842-2943-9 9

Reyes, J., Morales, L., Aldas, D., Reyes, R., \& Toasa, R. (2019). Real Time Automatic Andon Alerts for Android Platforms Applied in Footwear Manufacturing. In International Conference on Computer and Communication Engineering, pp. 43-56. https://doi.org/10.1007/978-3-030-12018-4 4

proyectosagiles. (s.f). https://proyectosagiles.org/que-es-scrum/

Silva, C. A., Toasa, R., Guevara, J., Martinez, H. D., \& Vargas, J. (2018). Mobile application to encourage local tourism with context-Aware computing. In International Conference on Information Technology \& Systems, ICITS'18, 796-803. https://doi.org/10.1007/978-3-319-73450-7 75 
Toasa, R., Silva, C., Silva, C., Goncalves, D., Neves, L., \& Marcelino, L. (2017). Energy consumption behaviour characterization with mobile gamification. In 2017 12th Iberian Conference on Information Systems and Technologies (CISTI) (pp. 1-6). https://doi.org/10.23919/CISTI.2017.7975900 


\section{Copyright (c) 2021 Josselyn Silvana Rodríguez Mejía}

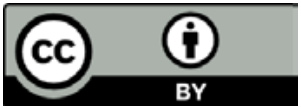

Este texto está protegido bajo una licencia internacional Creative Commons 4.0.

Usted es libre para Compartir-copiar y redistribuir el material en cualquier medio o formato - y Adaptar el documento - remezclar, transformar y crear a partir del material-para cualquier propósito, incluso para fines comerciales, siempre que cumpla las condiciones de Atribución. Usted debe dar crédito a la obra original de manera adecuada, proporcionar un enlace a la licencia, e indicar si se han realizado cambios. Puede hacerlo en cualquier forma razonable, pero no de forma tal que sugiera que tiene el apoyo del licenciante o lo recibe por el uso que hace de la obra.

Resumen de licencia - Texto completo de la licencia 